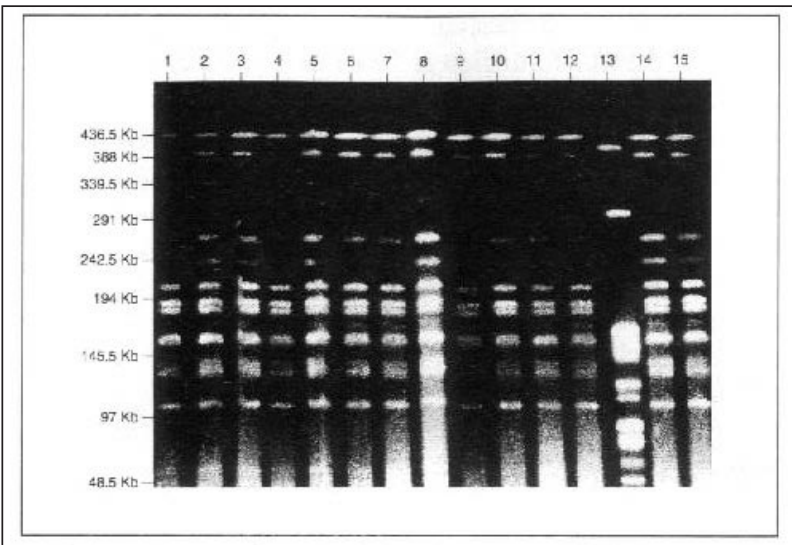

FIGURE. Pulsed-field gel electrophoresis of Apal chromosomal digests. All isolates are Acinetobacter haemolyticus except lane 13, which is Acinetobacter johnsonii. Lanes 1-9 and 11-15, patient isolates; Lane 10, phosphate-buffered saline isolate. Molecular weights of lambda concatamer marked on the side.

the several days in use to substantial numbers when mixed with the nasopharyngeal or endotracheal aspirates, and we have confirmed this experimentally. We have found that inoculation of phosphate-buffered saline with $10 \mathrm{CFU}$ of the outbreak strain results in multiplication to concentrations of $10^{6} \mathrm{CFU} / \mathrm{mL}$ within a week on incubation at room temperature. By contrast, we have found that Staphylococcus epidermidis similarly inoculated into phosphate-buffered saline fails to survive 24 hours' incubation at room temperature (unpublished observations).

The laboratory is a well-recognized source of pseudooutbreaks. Weinstein and $\mathrm{Stamm}^{4}$ found that 7 of 20 reported pseudo-outbreaks originated within the laboratory, usually following a change in personnel, technique, or culture medium. The first two factors were implicated in both of our pseudo-outbreaks, which was remarkable in that two independent errors in specimen processing occurred consecutively. The second pseudo-outbreak, caused by re-use of contaminated diluent, led to a false duplication of a variety of respiratory isolates, including the outbreak strain of Acinetobacter. This might have passed unnoticed but for the critical attitude to any increase in the isolation of Acinetobacter species fostered by the first pseu- do-outbreak. When the same specimen is handled by more than one department within the laboratory, an assessment of the implications of all aspects of specimen handling and processing is essential. Any change to agreed procedures also must be discussed between departments before implementation. Failure of such communication was responsible for the first pseudo-outbreak. The use of nonsterile Pasteur pipettes in the original virology bench protocol was appropriate for virological examination, but had not been agreed for bacteriology. This error was of no consequence for subsequent bacteriological examination, until the virology bench protocol was changed without authorization. The second outbreak was caused by a failure of communication between two bacteriology staff members sharing the same bench work, one of whom was new to the laboratory and not entirely familiar with its discard policy.

In conclusion, careful surveillance of culture results and patterns of isolation are essential for the prompt detection of pseudo-outbreaks. Thorough discussion and communication of agreed bench methodologies are essential to avoid pseudo-outbreaks originating within the laboratory.

96-CC-030. Address reprint requests to Hugo A. Ludlam, MD, Clinical Microbiology and Public Health Laboratory, Addenbrooke's Hospital, Cambridge CB2 2QW, England.

\section{REFERENCES}

1. Bergogne-Berezin E, Joly-Guillou ML, Vieu JF. Epidemiology of nosocomial infections due to Acinetobacter calcoaceticus. $J$ Hosp Infect 1987;10:105-113.

2. Snydman DR, Maloy MF, Brock SM, Lyons RW, Rubin SJ. Pseudobacteremia: false-positive blood cultures from mist tent contamination. Am J Epidemiol 1977;106:154-159.

3. Wilson PA, Petts DN, Baker SL. An outbreak of pseudobacteraemia. BMJ 1981;283:866.

4. Weinstein RA, Stamm WE. Pseudoepidemics in hospital. Lancet 1977;2:862-864.

5. Faris HM, Sparling FF. Mima polymorpha bacteremia. JAMA 1972;219:76-77.

6. Kaufmann ME, Pitt TL. Pulsed-field gel electrophoresis of bacterial DNA. In: Chart H, ed. Methods in Practical Laboratory Bacteriology. 1st ed. Boca Raton, FL: CRC Press; 1994:83-92.

7. Tenover FC, Arbeit RD, Georing RV, et al. Interpreting chromosomal DNA restriction patterns produced by pulsed-field gel electrophoresis: criteria for bacterial strain typing. J Clin Microbiol 1995;33:2233-2239.

\title{
Transmission of MDR TB From Inadequately Disinfected Bronchoscope
}

\section{Gina Pugliese, RN, MS Martin S. Favero, PhD}

Dr. Tracy Agerton and colleagues from the CDC recently assisted with an investigation in a hospital that reported four hospital-acquired cases of multidrug-resistant tuberculosis (MDR TB) with no community links. The investigation revealed that case 1 and case 4 both had clinical courses consistent with MDR TB, with many smear-negative and culture-positive specimens and cavitary lesions on chest radiograph; both died of MDR TB less than 1 month after diagnosis.

Case 2 had a skin-test conversion following bronchoscopy. Neither case 2 or case 3 had a clinical course consistent with MDR TB, neither was treated for MDR TB, and both remain alive and well. All four cases, however, had the same drug-resistance pattern, and the three available isolates had identical DNA fingerprints. No evidence of laboratory contamination of specimens, transmission on inpatient wards, or contact among cases was found. All of the cases received bronchoscopy in the same month. Cases 2, 3 , and 4 had their bronchoscopes done 1,12 , and 17 days, respectively, after case 1 . Observations revealed that bronchoscope cleaning was inade- quate, and the bronchoscope was never immersed in disinfectant.

The researchers concluded that inadequate cleaning and disinfection of the bronchoscope after case 1's procedure led to subsequent false-positive cultures in cases 2 and 3 , transmission of infection to case 2, and clinical MDR TB to case 4 .

FROM: Agerton TB, Valway S, Gore B, et al. Transmission of multidrug-resistant tuberculosis via bronchoscopy. Presented at the 46th Annual Epidemic Intelligence Service Conference; April 14-18, 1997; Atlanta, GA. 